*Note: This is an uncorrected version of an author's manuscript accepted for publication. ${ }^{*}$ Copyediting, typesetting, and review of the resulting proofs will be undertaken on this manuscript before final publication. During production and prepress, errors may be discovered that could affect the content.

\title{
Acute Cortisol Levels and Memory Performance in Older People with High and Normal Body Mass Index
}

Matias Pulopulos ${ }^{12}$, Sara Puig Pérez ${ }^{23}$, Vanesa Hidalgo ${ }^{24}$, Teresa Montoliu ${ }^{2}$, and Alicia Sal-

\author{
vador $^{2}$ \\ ${ }^{1}$ Universiteit Gent (Belgium) \\ ${ }^{2}$ Universitat de València (Spain) \\ ${ }^{3}$ Universidad Internacional de Valencia (Spain) \\ ${ }^{4}$ Universidad de Zaragoza (Spain)
}

We are grateful to Marta García Lluch, Dr. Carolina Villada, Dr. Mercedes Almela and Dr. Eva Lira for their support in the research process, and Ms. Cindy DePoy for the revision of the English text. This work was supported by the Spanish Ministerio de Educación y Ciencia (grants PSI2013/46889, FPU AP2010-1830 to M.M.P., FPI/BES-2008-004224 to V.H. and FPU12/04597 to S.P-P.), Generalitat Valenciana (grants PROMETEOII2015-20 and ISIC/2013/01), Universitat de València (grant UV-INV-PREDOC16F1-383576 to T.M.), Ghent University (Grant BOF 01P18916), and Government of Aragón, Department of Innovation, Research and University and FEDER “Construyendo desde Aragón”.

Correspondence concerning this article should be addressed to Matias Pulopulos. Universiteit Gent. Department of Experimental Clinical and Health Psychology. Henri Dunantlaan, 2. 9000 Gent (Belgium). E-mail: matias.pulopulos@ugent.be Phone: +32-092646472. 


\begin{abstract}
Previous studies have shown that healthy older adults may be less sensitive to the effects of acute cortisol levels on memory performance than young adults. Importantly, being overweight has recently been associated with an increase in both cortisol concentration and cortisol receptors in central tissues, suggesting that Body Mass Index (BMI) may contribute to differences in the relationship between memory and acute cortisol. This study investigates the role of BMI in the relationship between memory performance and acute cortisol levels in older people $(M=64.70$ years; $S D=4.24)$. We measured cortisol levels and memory performance (working memory and declarative memory) in 33 participants with normal (normal BMI = 18.50-24.99) and 36 participants with overweight BMI (overweight BMI $=25-29.99$ ). Overweight BMI participants showed worse performance on word-list learning $(p=.036,95 \%$ CI $\left.[0.08,2.18], \eta^{2}=0.07\right)$. Higher cortisol levels were related to higher proactive interference $(\beta=.364, p=.016,95 \%$ CI $[0.07,0.66])$, and BMI did not moderate any of the relationships investigated. In accordance with previous studies, our results show worse memory performance in individuals with overweight BMI. However, our results do not support the idea that memory performance in older people with higher BMI may be more sensitive to differences in acute cortisol levels than in older people with normal BMI. More research is needed to test this hypothesis with obese individuals $\left(\mathrm{BMI}>30 \mathrm{Kg} / \mathrm{cm}^{2}\right)$.
\end{abstract}

Keywords: body mass index, cortisol, memory, older people, overweight 
The aging process is associated with several changes in memory performance (Nyberg, Lövdén, Riklund, Lindenberger, \&nd Bäckman, 2012). Healthy older people may show a mild decrease in memory performance; however, no changes, or even an improvement, can also be observed in some older individuals (e.g., Rönnlund, Nyberg, Bäckman, \& Nilsson, 2005). Several studies have focused on investigating the factors that might explain this inter-individual variability. Weight status has been identified as a possible key factor that could at least partly contribute to differences in cognitive performance in older people (Chan et al., 2013). Along these lines, high Body Mass Index (BMI), defined as $25 \mathrm{~kg} / \mathrm{m}^{2}$ or greater (World Health Organization, 2018), has been associated with worse performance on hippocampus and prefrontal cortex-related memory tasks, such as declarative memory and working memory, in older people (e.g., Benito-León, Mitchell, Hernández-Gallego, \& Bermejo-Pareja, 2013; Gunstad, Lhotsky, Wendell, Ferrucci, \& Zonderman, 2010). However, the neurobiological mechanisms underlying this relationship are still unclear.

Several studies suggest that the activity of the Hypothalamic-Pituitary-Adrenal axis (HPA axis) and its end product, the hormone cortisol, might contribute to the effects of high BMI-related changes on memory performance (Lasikiewicz, Hendrickx, Talbot, \& Dye, 2013; Rotenstein, Sheridan, Garg, \& Adler, 2015; Sakata et al., 2012). It is a well-known fact that cortisol levels affect memory performance through the activation of the two types of cortisol receptors, the mineralocorticoid receptors (MRs) and the glucocorticoid receptors (GRs). Both receptors are especially located in the hippocampus, amygdala, and frontal cortex, making these structures very sensitive to cortisol levels (for a review see: Wolf et al., 2006). MRs have a higher affinity to cortisol than GRs, and they are especially responsible for the effects of cortisol on memory performance (de Kloet, Oitzl, \& Joëls, 1999). Given that the effects of cortisol on memory performance under non-stressful conditions depend on MRs, the same amount of cortisol would provoke a stronger effect in individuals with a higher density of MR. Previous studies focused on investigating the effects of cortisol levels have shown that high acute cortisol concentrations may affect memory performance. Specifically for endogenous cortisol levels during memory testing (what we refer to here as acute cortisol levels), higher acute cortisol levels have been related to worse short-term spatial memory performance (van Honk et al., 2003 ) and to better encoding and consolidation of emotional material (emotional faces and nonfaces pictures) recalled $20 \mathrm{~min}$ and $24 \mathrm{hr}$ later (Preuß, Schoofs, \& Wolf, 2009; Putman, van Honk, Kessels, Mulder, \& Koppeschaar, 2004) in young adults. Additionally, greater decreases in cortisol levels during memory testing were related to better performance on picture recall 24 hr after learning (Ackermann, Hartmann, Papassotiropoulos, de Quervain, \& Rasch, 2013). 
These studies demonstrated that differences in acute cortisol levels are related to differences in memory performance in young adults. Importantly, higher BMI $\left(\mathrm{BMI}>25 \mathrm{~kg} / \mathrm{m}^{2}\right)$ has been associated with higher cortisol levels (e.g., Pulopulos et al., 2014) and higher MR levels (Rotenstein et al., 2015; Sakata et al., 2012), suggesting that cortisol may at least partly explain the BMI-related differences in memory performance.

Studies in animals and humans suggest that overweight is associated with an increase in MR levels in central tissues (Rotenstein et al., 2015; Sakata et al., 2012). Moreover, they have shown that the administration of MR antagonists improves spatial memory in mice (Sakata et al., 2012) and the performance on a paired-associate learning task of pictures in individuals with obese BMI (Rotenstein et al., 2015). Given that the effect of cortisol on memory performance depends on the number of MR in the central nervous system, an increase in MR in individuals with higher BMI would enhance the sensitivity of this population to the effect of cortisol. In this direction, in a study investigating the effects of acute stress on memory performance, Lasikiewicz et al. (2013) observed that middle-aged people (age range between $35-65$, mean $=46 \pm 7.36$ ) with central obesity showed a stronger relationship between stressinduced increases in cortisol levels and worse performance on spatial recognition memory and paired-associate learning than middle-aged people without central obesity. Importantly, this idea has only been investigated in individuals with central obesity (i.e., waist to hip ratio greater than 0.85 in females and 0.90 in males) (Lasikiewicz et al., 2013), and with BMI higher than $30 \mathrm{Kg} / \mathrm{cm}^{2}$ (Rotenstein et al., 2015), but not in overweight individuals (BMI 25-29.9kg/m²). Moreover, it is important to note that stress provokes an acute increase in cortisol levels that is not observed under non-stressful conditions. Thus, the effects of cortisol on memory performance may be different when the relationship between acute cortisol and memory performance is investigated. Thus, in sum, BMI could be an important factor that moderates the impact of cortisol on brain areas responsible for memory performance, given its influence on MR cortisol receptors in central tissues.

It is important to note that, in contrast to what is observed in young adults, it has been shown that memory performance in older people could be less sensitive to differences in cortisol levels due to a decrease in the density and sensitivity of cortisol receptors in the central nervous system (e.g., Giordano et al., 2005). The evidence for this age-related decrease in sensitivity comes from studies investigating the effects of stress-induced and/or pharmacologicallyinduced acute increases in cortisol levels on memory performance (Hidalgo et al., 2015; Porter, Barnett, Idey, Mcguckin, \& O'brien, 2002; Yehuda, Harvey, Buchsbaum, Tischler, \& Schmeidler, 2007; Pulopulos et al., 2013; Pulopulos et al., 2015; Wolf et al., 2001). Moreover, 
two recent studies indicate that the relationship between cortisol levels under non-stressful conditions and memory performance in older people would only be observed in individuals who are carriers of the ApoE- $-\varepsilon 4$ allele, which is considered a high-risk factor for developing Alzheimer Disease (Lee et al., 2008; Montoliu et al., 2018). Given the BMI-related increase in $\mathrm{MR}$, it is critical to understand whether differences in BMI contribute to a different relationship between cortisol and memory performance in older people.

Taken together, results have shown that aging is associated with a reduction in the sensitivity to the effects of acute cortisol levels on memory. However, some evidence indicates that a high BMI may contribute to people's increased sensitivity to differences in acute cortisol levels due to BMI-related changes in MR. Importantly, the role of BMI in the relationship between acute cortisol levels and memory performance in older people has not been studied, and more research is still needed. Based on this idea, the main aim of this study was to investigate the relationship between memory performance and acute cortisol levels (i.e., cortisol levels immediately before the memory assessment), considering the moderator role of BMI in this relationship in older people. Given that obesity is associated with several health conditions that may affect cortisol and memory performance and, thus, influence our results (Nguyen, Magno, Lane, Hinojosa, \& Lane, 2008; Pulopulos Hidalgo, Puig-Perez, \& Salvador, 2016; Spauwen, Köhler, Verhey, Stehouwer, \& van Boxtel, 2013), in this first study, we investigated healthy older individuals with normal (BMI 18.50-24.99 kg/m2) and overweight BMIs (BMI $25-29.9 \mathrm{~kg} / \mathrm{m}^{2}$ ). We assessed declarative memory and working memory in 69 older participants, and we divided the sample according to their BMI (i.e., normal and overweight BMI). First, we explored differences in cortisol levels and memory performance between these two groups, and then we investigated the relationship between cortisol and memory performance. Given that obesity has been related to higher MR levels, the memory assessment was carried out in the morning when higher cortisol levels can be observed; thus, greater MR occupation was expected (Sapolsky, Romero, \& Munck, 2001).

Based on previous studies, we proposed the following hypotheses: (i) Higher cortisol levels and worse memory performance will be observed in older people with overweight BMI. (ii) Given that individuals with higher BMI are expected to have higher MR, the higher BMI population will show greater sensitivity to cortisol's effect on cognitive performance (Lasikiewicz et al., 2013; Rotenstein et al., 2015; Sakata et al., 2012). Therefore, even with similar cortisol levels, inter-individual differences in acute cortisol levels during the session will show a stronger relationship with memory performance in older people with higher BMIs than in normal BMI participants. Importantly, both positive and negative relationships between 
cortisol and memory performance have been observed, and so we do not predict the direction of the relationship between cortisol and memory in our study. It is important to note that, although similar results might be observed in young adults, in the current study, we focused on older people because several recent studies have shown that healthy older people may be less sensitive to cortisol effects on memory performance (for a review see Hidalgo, Pulopulos, \& Salvador, 2019). Therefore, this study will offer critical information about the influence of BMI on this age-related reduction in cortisol sensitivity.

\section{Method}

\section{Participants}

Participants belonged to a study program at the University of Valencia for people older than 55 years of age. The participants recruited for the present study are part of a larger project, and some subjects later participated in different experimental sessions to investigate the relationship between acute stress and cognitive performance (Hidalgo et al., 2015; Pulopulos et al., 2013; Pulopulos et al., 2015). The exclusion criteria for the current study were: smoking more than 10 cigarettes a day ${ }^{1}$; alcohol or other drug abuse; having visual or hearing problems; presence of cardiovascular (e.g., hypertension), neurological, endocrine (e.g., Type 2 Diabetes) disease or psychiatric disorders; using any medication directly related to emotional or cognitive functioning or capable of influencing hormonal levels, such as glucocorticoids, psychotropic substances, or sleep medications; having been under general anaesthesia once or more than once in the past year; and the presence of a stressful life event during the past year. A BMI $>30$ $\mathrm{kg} / \mathrm{m}^{2}$ is usually related to the development of important health problems, such as diabetes and cardiovascular diseases, which also contribute to a dysregulation of the HPA-axis activity and impairments in memory performance (Nguyen et al., 2008; Pulopulos et al., 2016; Spauwen et al., 2013). Then, to exclude possible confounders in our results, in this study we focused on older people with normal (BMI 18.50-24.99 kg/m²) and overweight BMIs (BMI 25$29.9 \mathrm{~kg} / \mathrm{m}^{2}$ ), following the World Health Organization criteria (World Health Organization, 2000). Based on these exclusion criteria, a subsample of 70 participants were considered for the current study. One participant was excluded because he did not provide a large enough salivary sample to measure cortisol levels, and so the final sample was composed of 69 participants (32 men and 37 women), with ages ranging from 56 to 77 years old $(M=63.76$; $S D=3.91)$.

\footnotetext{
${ }^{1}$ Three participants in the High-BMI group were smokers. The exclusion of these participants did not change the statistical conclusions of the study.
} 
We divided the sample into normal BMI (BMI $=18.50-24.99 ; 13$ men and 20 women $)$ and overweight $\mathrm{BMI}(\mathrm{BMI} \geq 25 ; 19$ men and 17 women). More than half of the participants had an educational level beyond high school (61\%). All the female participants were postmenopausal and had their last menstrual period more than three years before the testing time. None of these women were receiving estrogen replacement therapy. None of the participants scored below 28 on the Mini-Mental Status Examination (Lobo et al., 1999), indicating that there was no suspicion of cognitive impairment.

\section{Procedure}

Participants attended an individual session carried out at the Faculty of Psychology and starting at 10:00 hr or at 12:00 hr. Thirty-one participants started the session at 10:00 hr (High$\mathrm{BMI}=19$, Normal-BMI $=12)$, and 38 began at 12:00 hr (overweight BMI $=17$, normal BMI $=21)$. There were no significant differences in the number of participants in the groups that started the session at 10:00 $\mathrm{hr}$ or 12:00 hr, $\chi^{2}(1, N=69)=1.88, p=.17$. Before each session, participants were asked to sleep as much as usual, refrain from heavy physical activity the day before and the day of the session, and not drink alcohol since the night before the session. Furthermore, participants were instructed to drink only water and refrain from eating or consuming any stimulants (such as caffeine, cola, coffee, tea or chocolate) one hour before the session, or from brushing their teeth at least 1 hour prior to the session. The study was conducted according to the Declaration of Helsinki and approved by the Research Ethics Committee of the University of Valencia. All participants provided their written informed consent to participate in the study.

After their arrival at the laboratory, participants' weight and height were measured. Height was measured to the nearest $0.1 \mathrm{~cm}$ without footwear. Weight was measured to the nearest $0.1 \mathrm{~kg}$ using a Soehnle Professional medical scale (Soehnle Industrial Solutions GmbH, Backnang, Germany). Next, the participants remained seated for $15 \mathrm{~min}$ to become habituated to the laboratory while they filled out questionnaires. Then, the participants provided one saliva sample to measure acute cortisol levels. Finally, they performed the Mini-Mental Status Examination and the memory tasks to assess declarative memory and working memory.

\section{Demographics and physical activity}

Participants filled out a questionnaire on age, subjective socioeconomic status (SES; measured using the MacArthur Scale of Subjective Social Status; Adler, Epel, Castellazzo, \& Ickovics, 2000), educational level ( 0 = no studies; 1 = primary school; 2 = secondary education; $3=$ Bachelor and master education; $4=$ Doctorate $)$, and weekly physical activity $(0=$ none $; 1$ $=$ low $; 2=$ moderate $; 3=$ high). 


\section{Cortisol measurements}

Salivary samples were collected using salivettes (Sarstedt, Nümbrecht, Germany). Salivary cortisol levels were measured in duplicate by a competitive solid phase radioimmunoassay (tube coated) using the commercial kit Spectria Cortisol RIA from Orion Diagnostica (Espoo, Finland). Assay sensitivity was $0.8 \mathrm{nmol} / \mathrm{L}$, and the within- and inter-assay variation coefficients were all below $8 \%$.

\section{Memory Assessment}

\section{Declarative memory test}

The Rey Auditory Verbal Learning Test (RAVLT; Rey, 1958) was used to assess declarative memory. This test is composed of eight trials. In the first five trials, the experimenter read aloud a list of 15 neutral nouns (List A), and participants had to repeat as many words as possible from list A after each of the five trials. After Trial 5, the experimenter read aloud a different list of 15 words (List B, Trial 6) and tested the recall of these new words. Then, immediately after recalling List B, the participants were asked to recall the words from list A (Trial 7). After a delay of 20 min, they had to recall list A again (Trial 8). List A and List B were always the same for all the participants.

Five indexes were calculated for this test: (i) Learning: The number of words recalled in Trial 5 minus the number of words recalled in Trial 1 was used as an index of learning performance; (ii) immediate recall: Number of words recalled in Trial 7; (iii) delayed recall: Percentage of words recalled after the 20-min delay (Trial 8) compared to the words recalled in the immediate recall (Trial 7); (iv) proactive interference: The number of words recalled in Trial 1 minus the number of words recalled from the interference list; (v) retroactive interference: the number of words recalled in Trial 5 minus the number of words recalled in Trial 7. For proactive interference and retroactive interference, higher scores are indicators of worse performance.

\section{Working memory test}

The Digit Span Forward (DS-Forward) and Digit Span Backward (DS-Backward) from the Wechsler Memory Scale III (Wechsler, 1997) were used to measure working memory. The DS-Forward was used as a measure of the memory span component of working memory. Participants repeated the sequence of digits in the same order. This part contains 8 sets, with two trials for each of these sets, and possible scores ranged from 0 to 16 .

The DS-Backward was used as a measure of the executive component of working memory. Participants repeated the sequence of digits in the reverse order. This part consists of 7 sets with two trials for each set. Possible scores ranged from 0 to 14 . The DS-Backward is a 
task that contains both a memory span component (as assessed with the DS-Forward) and an executive component (manipulating numbers in memory). Then, in all the analyses with DSBackward, we included DS-Forward as covariate to pinpoint the executive function of manipulating numbers in memory.

\section{Statistical analyses and data management}

Kolmogorov-Smirnov and Levene's tests revealed significant deviations in cortisol levels; therefore, they were sqrt transformed.

Differences between the normal BMI and overweight BMI groups were explored using Student's $t$-test (for age, SES and BMI), the Chi-squared test (for educational level, sex, and physical exercise) and ANCOVA (for RAVLT indexes, Digit Span and cortisol levels with Age, SES, weekly physical activity and time of the session as covariates). Additionally, specifically for DS-Backward, DS-Forward was included as covariate. To investigate whether the relationship between cortisol and memory performance is stronger in individuals with higher BMI, moderated regression analyses using PROCESS for SPSS (Model 1) were performed. We included Age, SES, time of the session, and weekly physical activity (and DSForward for the analysis with DS-Backward) as covariates, cortisol levels as the predictor, and BMI as the moderator. The covariates were included in the ANCOVAS and regression analyses to control for their possible effect on memory performance and cortisol levels (e.g., Chan et al., 2013; Dettenborn, Tietze, Kirschbaum, \& Stalder, 2012; Wright \& Steptoe, 2005)².

Two outliers in the cortisol data (two participants in the normal BMI group) and two outliers in the Delayed recall data (two participants in the normal BMI group) were removed from the analyses because their outcomes differed by more than $3.0 S D$ from the total sample mean.

\section{Results}

\section{Characteristics of the sample}

Table 1 shows the characteristics of the groups. The overweight BMI group showed a significantly higher BMI than the normal BMI group, $t(67)=11.10,95 \%$ CI $[2.868,4.125], p<$ .001). There were no significant differences between the Normal-BMI and overweight-BMI groups on time of the session, age, SES, educational level, and weekly physical activity (all $p$ $>.119)$.

\footnotetext{
${ }^{2}$ The statistical conclusions of the study remain the same if all the analyses are performed without covariates.
} 


\section{$<$ Insert Table 1 Here $>$}

\section{Differences in memory performance and cortisol levels}

Table 1 shows the differences in memory performance and cortisol levels. The overweight BMI group showed worse performance on learning than the normal BMI group, $F(1,61)=4.62,95 \%$ CI $[0.08,2.18], \eta^{2}=0.07, p=.036$. No significant differences were observed for the other RAVLT indexes, Digit Span Forward, Digit Span Backward or cortisol levels (all $p>.121)$.

\section{$<\mathrm{H} 2>$ Relationship between cortisol and memory performance}

The moderated regression analyses showed that high acute cortisol levels were related to worse proactive interference, as per confidence intervals, $R^{2}=.12, \beta=.364, p=.016,95 \%$ CI $[0.07,0.66]$. None of the other relationships between cortisol and memory outcomes for whole group were statistically significant, according to confidence intervals or $p$-values (RAVLT: Learning: $R^{2}=.07, \beta=-.147, p=.352,95 \%$ CI [-0.46, 0.17]; immediate recall: $R^{2}$ $=.05, \beta=-.100, p=.506,95 \%$ CI $[-0.40,0.20]$; retroactive interference: $R^{2}=.06, \beta=-.123$, $p=.400,95 \%$ CI $[-0.43,0.17]$; delayed recall: $R^{2}=.15, \beta=-.193, p=.177,95 \%$ CI $[-0.48$, 0.09]; digit span test: Digit Span Forward: $R^{2}=.12, \beta=.041, p=.780,95 \%$ CI $[-0.25,0.33]$; Digit Span Backward: $R^{2}=.65, \beta=-.009, p=.947,95 \%$ CI [-0.26, 0.24]). Moreover, the confidence intervals indicate that BMI was not related to any of the memory outcomes (RAVLT: Learning: $\beta=-.106, p=.463,95 \%$ CI $[-0.39,0.18]$; immediate recall: $\beta=-.190, p$ $=.170,95 \% \mathrm{CI}[-0.46,0.08]$; proactive interference: $\beta=-.174, p=.200,95 \% \mathrm{CI}[-0.44,0.09]$; retroactive interference: $\beta=.090, p=.516,95 \% \mathrm{CI}[-0.18,0.36]$; delayed recall: $\beta=-.082, p$ $=.531,95 \%$ CI $[-0.34,0.18]$; digit span test: Digit Span Forward: $\beta=.156, p=.248,95 \%$ CI $[-0.11,0.42]$; Digit Span Backward: $\beta=-.068, p=.562,95 \%$ CI $[-0.30,0.17])$. Finally, the analyses showed that BMI does not moderate any of the relationships between cortisol and the memory outcomes (all $p>.400$ ).

\section{$<$ Insert Figure 1 Here $>$}

\section{Discussion}

This study investigated the differences in acute cortisol levels and memory performance and the relationship between cortisol levels and memory performance in older people with normal and overweight BMIs. Results showed worse performance on learning in older people with overweight BMI than in older people with normal BMI. No significant differences were observed in cortisol levels. Additionally, we observed that higher cortisol levels were related to worse proactive interference in the whole sample, and, in contrast to our hypothesis, BMI did 
not moderate the relationship between cortisol and memory performance.

We observed that older people with overweight BMI performed worse on word learning than people with normal BMI. Our results agree with previous studies showing worse memory performance in individuals with overweight and obese BMI (e.g., Benito-León et al., 2013; Chan et al., 2013; Gunstad et al., 2010). It should be noted that, although the effect size of this difference was small-medium (i.e., $\eta^{2}$ p $=0.07$ ), this study included only healthy participants with normal and overweight BMI. Moreover, this difference was independent from weekly physical activity, a factor that can modulate the effect of BMI on cognitive performance in older people (Chan et al., 2013). Therefore, our results indicate that, even in healthy older adults, and controlling for important confounders, BMI explains part of the variance in the differences in cognitive performance, and this effect would be expected to be stronger in individuals with higher BMI scores (i.e., BMI $>30 \mathrm{~kg} / \mathrm{m}^{2}$ ). In contrast to previous research, we did not observe a significant difference in cortisol levels between groups (Bose, Oliván, \& Laferrère, 2009). One possible explanation for the lack of cortisol differences may be related to the cortisol assessment. In our study, we measured cortisol levels using only one salivary sample immediately before the memory assessment. Previous studies that have observed a clear relationship between high $\mathrm{BMI}\left(\mathrm{BMI}>25 \mathrm{~kg} / \mathrm{m}^{2}\right)$ and higher cortisol levels have measured cortisol levels throughout the day (using several salivary samples) or even in the previous month (using hair samples) (e.g., Bose et al., 2009; Pulopulos et al., 2014). Therefore, it is possible that more salivary samples are needed to observe cortisol differences.

Importantly, although no differences in cortisol levels were observed, when exploring the relationship between cortisol and memory performance, we observed that higher cortisol levels immediately before memory testing were related to worse proactive interference. Previous studies in young adults have also observed a relationship between acute cortisol and memory performance. For instance, van Honk et al. (2003), showed that higher acute cortisol levels were related to worse short-term spatial memory performance. Moreover, Ackermann et al. (2013) showed that a greater decrease in cortisol levels (i.e., lower cortisol levels) during memory recall was related to better performance on picture recall $24 \mathrm{hr}$ after learning. Although our results support these previous studies, it should be noted that the rate of change of the conditional mean of proactive interference values with respect to cortisol levels is estimated to be between 0.07 and 0.66 (standardized beta values). In addition to the wide range of confidence intervals, due to the number variables investigated and the number of analyses performed, a type II error should also be considered. Together, our results indicate that, in healthy older people, acute cortisol levels are only related, if at all, to Proactive Interference, but not to 
working memory, short-term memory, learning, and long-term memory. The lack of relationships between cortisol and most memory processes coincide with recent studies suggesting that healthy older people and animals may be less sensitive to acute effects of cortisol levels on memory performance (Buechel et al., 2014; Hidalgo et al., 2015; Porter et al., 2002; Pulopulos et al., 2013; Pulopulos et al., 2015; Yehuda et al., 2007; see Hidalgo et al., 2019 for a review). Thus, our results highlight the relevance of considering age as a key factor in understanding the differences in the effect of cortisol on memory performance.

We hypothesized that acute cortisol levels during the session would show a stronger relationship with memory performance in older people with higher BMIs than in normal BMI participants. In contrast with this proposal, BMI did not moderate the relationship between cortisol and memory performance. These results do not agree with previous studies showing that obesity increases sensitivity to the effects of cortisol levels on memory in humans (Lasikiewicz et al., 2013; Rotenstein et al., 2015) and animals (Sakata et al., 2012). Lasikiewicz et al. (2013) investigated the role of central obesity in the effect of acute stress on memory performance in middle-aged people (age range between $35-65$; mean $=46 \pm 7.36$ ). These authors observed a stronger relationship between stress-induced increases in cortisol levels and worse memory performance in middle-aged people with central obesity than in people without central obesity. Three methodological differences may explain the contradictory results between our study and a previous study in humans. First, given that cortisol levels after stress are higher than under non-stressful conditions, it is possible that higher cortisol levels are needed to observe an effect, as found in Lasikiewicz et al. (2013). In the current study, we did not manipulate the cortisol levels (e.g., using a stress task). Instead, we measured acute cortisol levels, which could provoke less variability in cortisol levels and reduce the possibility of observing significant associations between cortisol and memory performance. Second, the samples included in Lasikiewicz et al. (2013) and Rotenstein et al. (2015) were younger (age range: $35-65$, mean $=46.00$ and age range: $20-61$, mean $=44.13$, respectively) than the sample in our study. It is possible that the effect of BMI is evident in younger samples, but not in older samples, due to the age-related decrease in MR. Finally, it is important to note that the BMI of the participants in our study sample ranged from normal to overweight (BMI from 18 to 29.9 $\mathrm{kg} / \mathrm{m}^{2}$ ). However, Lasikiewicz et al. (2013) and Rotenstein et al. (2015) investigated participants with central obesity (i.e., waist to hip ratio greater than 0.85 in females and 0.90 in males) and BMI higher than $30 \mathrm{~kg} / \mathrm{m}^{2}$, respectively. Therefore, it is possible that differences in the relationship between cortisol and memory can only be observed in obese individuals (BMI $>30$ ). Taken together, our study indicates that, in healthy older people (age range between 56- 
77), the effect of overweight on memory performance might not be due to differences in MR.

A limitation of the current study is that, to avoid possible confounding factors, we excluded older people with some health problems commonly related to overweight and obesity (e.g., Type 2 Diabetes, Hypertension) that could affect the activity of the HPA-axis and/or memory performance. Thus, it is possible that larger differences in memory performance (e.g., Digit Span tasks and/or immediate recall) or cortisol levels might be observed if participants with high BMI-related diseases are included. Although this approach reduces the generalizability of our results, it allows us to better understand the role of BMI in the relationship between cortisol and memory. Another limitation of the study is that we did not control the time of awakening of our participants on the day of the session. Hence, the lack of differences in cortisol levels might be explained by differences in the time of awakening (e.g., Pulopulos et al., 2016).

In conclusion, our results confirm findings from previous studies showing worse memory performance in overweight people. Additionally, our study suggests that acute cortisol levels may contribute, at least in part, to interindividual differences in proactive interference in healthy older people, regardless of their BMI. Finally, our results suggest that the differences in memory performance between healthy older individuals with high and normal BMI are not due to a between-group difference in the relationship between acute cortisol levels and memory performance. 


\section{References}

Ackermann, S., Hartmann, F., Papassotiropoulos, A., de Quervain, D. J. F., \& Rasch, B. (2013). Associations between basal cortisol levels and memory retrieval in healthy young individuals. Journal of Cognitive Neuroscience, 25(11), 1896-1907. https://doi.org/10.1162/jocn a 00440

Adler, N. E., Epel, E. S., Castellazzo, G., \& Ickovics, J. R. (2000). Relationship of subjective and objective social status with psychological and physiological functioning: Preliminary data in healthy, white women. Health Psychology, 19(6), 586-592. http://doi.org/10.1037/02786133.19.6.586

Benito-León, J., Mitchell, A. J., Hernández-Gallego, J., \& Bermejo-Pareja, F. (2013). Obesity and impaired cognitive functioning in the elderly: A population-based cross-sectional study (NEDICES). European Journal of Neurology, 20(6), 899-906. http://doi.org/10.1111/ene.12083

Bose, M., Oliván, B., \& Laferrère, B. (2009). Stress and obesity: The role of the hypothalamic-pituitary-adrenal axis in metabolic disease. Current Opinion in Endocrinology, Diabetes and Obesity, 16(5), 340-346. http://doi.org/10.1097/med.0b013e32832fa137

Buechel, H. M., Popovic, J., Staggs, K., Anderson, K. L., Thibault, O., \& Blalock, E. M. (2014). Aged rats are hypo-responsive to acute restraint: Implications for psychosocial stress in aging. Frontiers in Aging Neuroscience, 6, 13. http://doi.org/10.3389/fnagi.2014.00013

Chan, J. S. Y., Yan, J. H., \& Payne, V. G. (2013). The impact of obesity and exercise on cognitive aging. Frontiers in Aging Neuroscience, 5, article 97. http://doi.org/10.3389/fnagi.2013.00097

de Kloet, E. R., Oitzl, M. S., \& Joëls, M. (1999). Stress and cognition: Are corticosteroids good or bad guys? Trends in Neurosciences, 22(10), 422-426. http://doi.org/10.1016/s0166$\underline{2236(99) 01438-1}$

Dettenborn, L., Tietze, A., Kirschbaum, C., \& Stalder, T. (2012). The assessment of cortisol in human hair: Associations with sociodemographic variables and potential confounders. Stress, 15(6), 578-588. http://doi.org/10.3109/10253890.2012.654479

Giordano, R., Bo, M., Pellegrino, M., Vezzari, M., Baldi, M., Picu, A., ... Arvat, E. (2005). Hypothalamus-pituitary-adrenal hyperactivity in human aging is partially refractory to stimulation by mineralocorticoid receptor blockade. The Journal of Clinical Endocrinology \& Metabolism, 90(10), 5656-5662. http://doi.org/10.1210/jc.2005-0105

Gunstad, J., Lhotsky, A., Wendell, C. R., Ferrucci, L., \& Zonderman, A. B. (2010). Longitudinal examination of obesity and cognitive function: Results from the Baltimore Longitudinal Study of Aging. Neuroepidemiology, 34(4), 222-229. http://doi.org/10.1159/000297742

Hidalgo, V., Pulopulos, M. M., Puig-Perez, S., Espin, L., Gomez-Amor, J., \& Salvador, A. (2015). Acute stress affects free recall and recognition of pictures differently depending on age and sex. Behavioural Brain Research, 292, 393-402.

Pulopulos, M. M., \& Salvador, V., Pulopulos, M. M., \& Salvador, A. (2019). Acute psychosocial stress effects on memory performance: Relevance of age and sex. Neurobiology of Learning and Memory, 157, 48-60. https://doi.org/10.1016/j.nlm.2018.11.013

Lasikiewicz, N., Hendrickx, H., Talbot, D., \& Dye, L. (2013). Exploring stress-induced cognitive impairment in middle aged, centrally obese adults. Stress, 16(1), 44-53. http://doi.org/10.3109/10253890.2012.682109

Lee, B. K., Glass, T. A., Wand, G. S., Mcatee, M. J., Bandeen-Roche, K., Bolla, K. I., \& Schwartz, B. S. (2008). Apolipoprotein E genotype, cortisol, and cognitive function in community-dwelling older adults. The American Journal of Psychiatry, 165(11), 1456-1464. https://doi.org/10.1176/appi.ajp.2008.07091532

Lobo, A., Saz, P., Marcos, G., Día, J. L., De La Cámara, C., Ventura, T., ... Aznar, S. (1999). [Revalidation and standardization of the cognition mini-exam (first Spanish version of the Mini-Mental Status Examination) in the general geriatric population]. Medicina Clínica, 112(20), 767774.

Montoliu, T., Hidalgo, V., Pulopulos, M. M., Ivorra, J. L., Martínez, M. J., \& Salvador, A. (2018). The relationship between cortisol and cognitive function in healthy older people: The moderating role of Apolipoprotein E polymorphism. Neurobiology of Learning and Memory, 155, 297-305. https://doi.org/10.1016/j.nlm.2018.08.013

Nguyen, N. T., Magno, C. P., Lane, K. T., Hinojosa, M. W., \& Lane, J. S. (2008). Association of hypertension, diabetes, dyslipidemia, and metabolic syndrome with obesity: findings from the 
National Health and Nutrition Examination Survey, 1999 to 2004. Journal of the American College of Surgeons, 207(6), 928-934. https://doi.org/10.1016/j.jamcollsurg.2008.08.022

Nyberg, L., Lövdén, M., Riklund, K., Lindenberger, U., \& Bäckman, L. (2012). Memory aging and brain maintenance. Trends in Cognitive Sciences, 16(5), 292-305. https://doi.org/10.1016/j.tics.2012.04.005

Porter, R. J., Barnett, N. A., Idey, A., Mcguckin, E. A., \& O'brien, J. T. (2002). Effects of hydrocortisone administration on cognitive function in the elderly. Journal of Psychopharmacology, 16(1), 65-71. http://doi.org/10.1177/026988110201600106

Preuß, D., Schoofs, D., \& Wolf, O. T. (2009). Associations between endogenous cortisol levels and emotional memory in young women: Influence of encoding instructions. Stress, 12(5), 379387. https://doi.org/10.1080/10253890802524592

Pulopulos, M. M., Almela, M., Hidalgo, V., Villada, C., Puig-Perez, S., \& Salvador, A. (2013). Acute stress does not impair long-term memory retrieval in older people. Neurobiology of Learning and Memory, 104, 16-24. http://doi.org/10.1016/j.nlm.2013.04.010

Pulopulos, M. M., Hidalgo, V., Almela, M., Puig-Perez, S., Villada, C., \& Salvador, A. (2014). Hair cortisol and cognitive performance in healthy older people. Psychoneuroendocrinology, 44, 100-111. http://doi.org/10.1016/j.psyneuen.2014.03.002

Pulopulos, M. M., Hidalgo, V., Almela, M., Puig-Perez, S., Villada, C., \& Salvador, A. (2015). Acute stress and working memory in older people. Stress, 18(2), 178-187. http://doi.org/10.3109/10253890.2015.1004538

Pulopulos, M. M., Hidalgo, V., Puig-Perez, S., \& Salvador, A. (2016). Cortisol awakening response and cognitive performance in hypertensive and normotensive older people. Hormones and Behavior, 83, 75-82. http://doi.org/10.1016/j.yhbeh.2016.05.014

Putman, P., van Honk, J., Kessels, R. P., Mulder, M., \& Koppeschaar, H. P. (2004). Salivary cortisol and short and long-term memory for emotional faces in healthy young women. Psychoneuroendocrinology, 29(7), 953-960. http://doi.org/10.1016/j.psyneuen.2003.09.001

Rey, A. (1958). L'examen clinique en psychologie [The clinical exam in psychology]. Paris, France: Presse Universitaire de France.

Rönnlund, M., Nyberg, L., Bäckman, L., \& Nilsson, L.-G. (2005). Stability, growth, and decline in adult life span development of declarative memory: Cross-sectional and longitudinal data from a population-based study. Psychology and Aging, 20(1), 3-18. http://doi.org/10.1037/08827974.20.1.3

Rotenstein, L. S., Sheridan, M., Garg, R., \& Adler, G. K. (2015). Effect of mineralocorticoid receptor blockade on hippocampal-dependent memory in adults with obesity. Obesity, 23(6), 11361142. http://doi.org/10.1002/oby.21104

Sakata, A., Mogi, M., Iwanami, J., Tsukuda, K., Min, L.J, Jing, F., ... Horiuchi, M. (2012). Improvement of cognitive impairment in female type 2 diabetes mellitus mice by spironolactone. Journal of the Renin-Angiotensin-Aldosterone System, 13(1), 84-90. http://doi.org/10.1177/1470320311412810

Sapolsky, R. M., Romero, L. M., \& Munck, A. U. (2001). How do glucocorticoids influence stress responses? Integrating permissive, suppressive, stimulatory, and preparative actions. Endocrine Reviews, 21, 55-89. https://doi.org/10.1210/edrv.21.1.0389

Spauwen, P. J., Köhler, S., Verhey, F. R., Stehouwer, C. D., \& van Boxtel, M. P. (2013). Effects of Type 2 Diabetes on 12-year cognitive change: Results from the Maastricht Aging Study. Diabetes Care, 36(6), 1554-1561. http://doi.org/10.2337/dc12-0746

van Honk, J., Kessels, R. P., Putman, P., Jager, G., Koppeschaar, H. P., \& Postma, A. (2003). Attentionally modulated effects of cortisol and mood on memory for emotional faces in healthy young males. Psychoneuroendocrinology, 28(7), 941-948. http://doi.org/10.1016/s03064530(02)00116-6

Wechsler, D. (1997). The Wechsler Memory Scale ( $3^{\text {rd }}$ Ed.). San Antonio, TX: Psychological Corporation.

Wolf, O. T., Convit, A., Mchugh, P. F., Kandil, E., Thorn, E. L., De Santi, S., ... de Leon, M. J. (2001). Cortisol differentially affects memory in young and elderly men. Behavioral Neuroscience, 115(5), 1002-1011. http://doi.org/10.1037//0735-7044.115.5.1002 
Wolf, O. T. (2006). Effects of stress hormones on the structure and function of the human brain. Expert Review of Endocrinology \& Metabolism, 1(5), 623-632. http://doi.org/10.1586/17446651.1.5.623

World Health Organization (2000). Obesity: Preventing and managing the global epidemic. Geneva, Switzerland: World Health Organization.

World Health Organization (2018) Obesity and overweight. Retrieved from World Health Organization website. http://www.who.int/news-room/fact-sheets/ detail/obesity-and-overweight

Wright, C. E., \& Steptoe, A. (2005). Subjective socioeconomic position, gender and cortisol responses to waking in an elderly population. Psychoneuroendocrinology, 30(6), 582-590. http://doi.org/10.1016/j.psyneuen.2005.01.007

Yehuda, R., Harvey, P. D., Buchsbaum, M., Tischler, L., \& Schmeidler, J. (2007). Enhanced effects of cortisol administration on episodic and working memory in aging veterans with PTSD. Neuropsychopharmacology, 32(12), 2581-2591. http://doi.org/10.1038/sj.npp.1301380 
Table 1.

Participants' Characteristics and Memory Performance

\begin{tabular}{lcc}
\hline & $\begin{array}{c}\text { High-BMI } \\
(M \pm S D)\end{array}$ & $\begin{array}{c}\text { Normal-BMI } \\
(M \pm S D)\end{array}$ \\
\cline { 2 - 3 } Age (years) & $64.86 \pm 3.60$ & $64.15 \pm 4.43$ \\
BMI $\left(\mathrm{kg} / \mathrm{m}^{2}\right)$ & $26.93 \pm 1.34$ & $23.43 \pm 1.27$ \\
SES & $5.86 \pm 1.23$ & $5.45 \pm 1.00$ \\
Educ. Level & $2.58 \pm 0.77$ & $2.33 \pm 0.99$ \\
Physical activity & $1.89 \pm 0.75$ & $1.91 \pm 0.77$ \\
DS-Forward & $8.86 \pm 2.00$ & $8.37 \pm 1.84$ \\
DS-Backward & $6.25 \pm 1.90$ & $5.75 \pm 2.10$ \\
RAVLT Learning & $4.86 \pm 2.14$ & $5.87 \pm 1.94$ \\
RAVLT PI & $1.97 \pm 1.97$ & $1.64 \pm 2.39$ \\
RAVLT RI & $1.36 \pm 1.55$ & $1.35 \pm 1.87$ \\
RAVLT Immed. & $10.36 \pm 2.17$ & $11.03 \pm 2.50$ \\
RAVLT Delayed & $99.31 \pm 11.12$ & $98.88 \pm 9.80$ \\
Cortisol (nmol/L) & $6.93 \pm 3.06$ & $6.00 \pm 3.41$ \\
\hline Note. BMI = Body Mass Index; SES $=$ Subjective \\
socioeconomic status; Educ. Level = Educational level; DS $=$ \\
Digit Span; RAVLT = Rey Auditory Verbal Learning Test; \\
PI = Proactive Interference; RI = Retroactive Interference; \\
Immed. = Immediate.
\end{tabular}




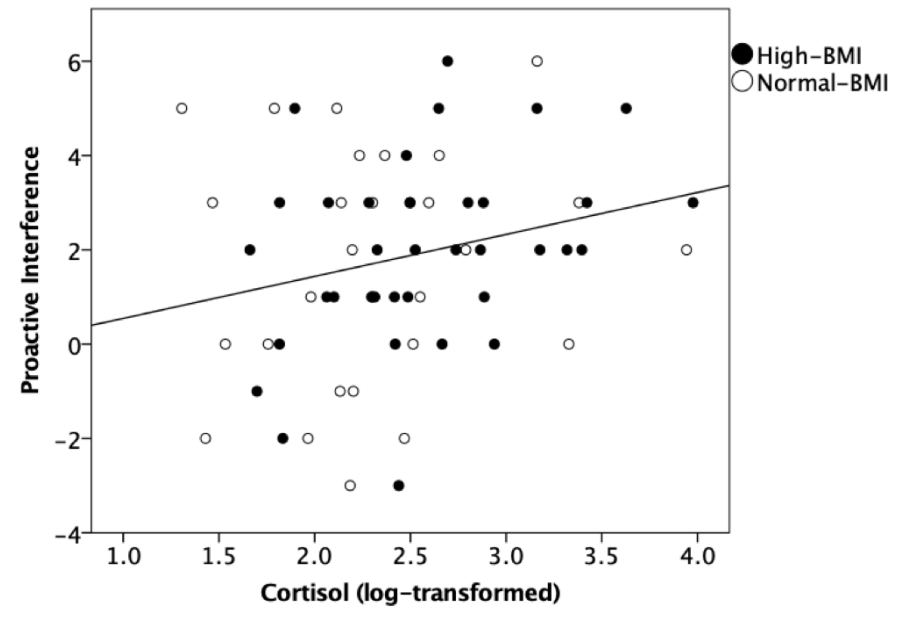

Figure 1. Relationship between Acute Cortisol Levels and Proactive Interference in Participants with Overweight BMI and Normal BMI. The regression analyses showed that high acute cortisol levels were related to worse proactive interference, $R^{2}=.12, \beta=.364, p=$ $.016,95 \%$ CI [0.07, 0.66]. 